crystal structure of lanthanum iron oxide (perovskite structure) has a long axis that lay in the plane of the thin-film sample along two directions at right angles. Both the size and orientation of the sample's crystal domains coincided with its magnetic domains, showing that they are closely correlated.

Lanthanum iron oxide is an antiferromagnetic material whose domain structure is large enough to be resolved by the PEEM2, but it is not the material used in technological devices. Eric Fullerton of IBM Almaden Research Center said that "in current read-head devices, more common antiferromagnets like nickel oxide and iron manganese are used." He said that the study of those materials will require higher resolution.

\section{Graphite Fugitive Layer Assists Formation of Nonporous Zirconia Layer on Fuel-Cell Tubes}

Solid-oxide fuel cells convert gaseous, hydrogen-rich fuels like natural gas, biogas, alcohols, and coal-derived gas directly into electrical energy. They do this in a reaction that eventually breaks the fuel down into water and carbon dioxide. The tubular solid-oxide fuel cells, consisting of bundles of tubes with oxygen flowing inside and gaseous hydrocarbon flowing over the tubes, operates at about $1800^{\circ} \mathrm{F}$. Working with Merrilea J. Mayo and Clive A. Randall, both associate professors of materials science and engineering at The Pennsylvania State University, postdoctoral associate Rajendra N. Basu developed a method to apply a gas-tight layer of zirconia on the tubes. Along with separating the fuel from the air in order to avoid an explosion, zirconia serves as a conductor of oxygen ions.

Mayo said, "Electricity is produced by the oxygen gas-to-oxygen ion interconversion, which occurs at the surfaces of the zirconia film: On the air-exposed surface, oxygen gas separates into oxygen ions. The ions travel through the film, then give up their electrons on the other side when they react with hydrogen to form water. The electrons thus generated are captured in an external circuit, to provide an electricity source."

Basu, who is a scientist at Central Glass and Ceramic Research Institute, a national laboratory in Calcutta, India, said that other researchers have tried using electrophoretic deposition to make zirconia coating, "but with limited success." While electrochemical vapor deposition is used to make these coatings, the existing method is very expensive, driving up the costs of manufacturing solid oxide fuel cells.

In electrophoretic deposition, a suspension of yttrium-doped zirconium oxide powder is made in very high concentration acetic acid. The application of an electrical potential allows the charged powder to move toward and deposit on the electrode with the opposite charge. The object with its powder coating is then fired at a very high temperature so that the coating forms into a continuous film on the underlying material.

However, depositing zirconium oxide on the bare, porous-ceramic cathode-tube surfaces of the tubular solid-oxide fuel cells leads to an inhomogeneous coating that is not gas-tight. The researchers deduced that the pores of the cathode tube were the source of the problem and tried a fugitive layer of carbon between the tube and the coating. This graphite layer serves as a uniform cathode, and the zirconium oxide deposits evenly on the surface. During firing, the graphite sublimes and the coating deposits evenly on the tube's surface.

"We achieved a very even, homogeneous layer without any porosity," said

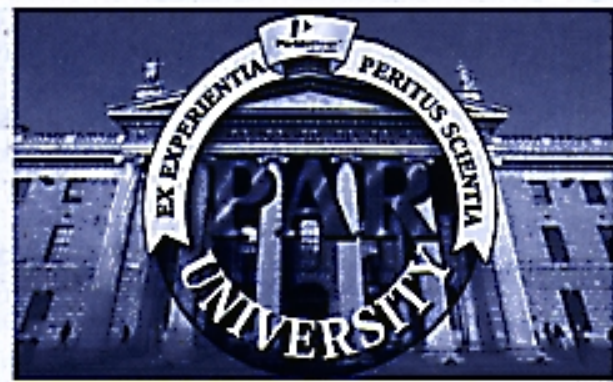

www.par-online.com

More than just our name has changed.

Shop online beginning March 2000.

Enroll in our training courses through PAR University.

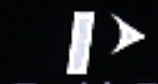

PerkinElmer

801 South Illinois Arenue Oak Ridge. TN 37831-0895

phone: (B65) 482-4411 ext 345

Ioll-Iree: $800 \cdot 366-274$ Iax: (865) $425 \cdot 1334$

\section{STOP. SHOP. LEARN.}

Princeton Applied Research is your complete source for instrumentation and training for all your Materials Characterization needs.

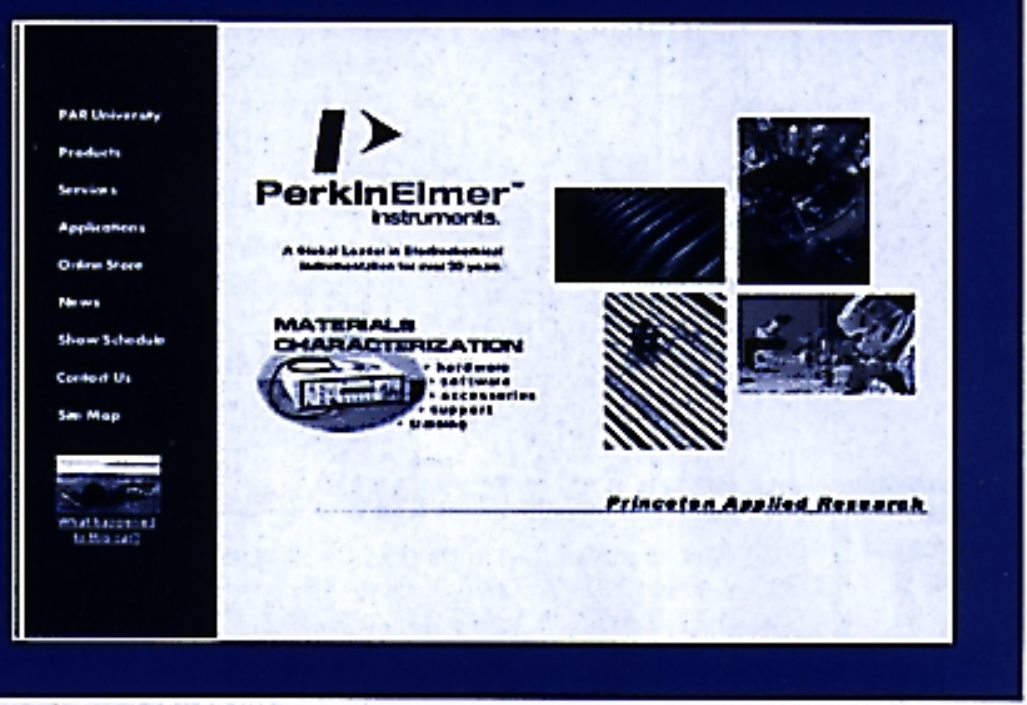

Circle No. 21 on Inside Back Cover 
Basu. "We can also easily adapt this process to coating long tubes."

\section{Simulations Reveal Morphological Transition in Simple Foams}

By deriving an equation of state for compressible foam and then simulating it numerically, researchers at the University of Illinois predict a dramatic morphological change that will occur as the surface tension is increased or, equivalently, the volume of the foam is greatly expanded. Foams are ubiquitous in nature and wide- ly used in industry, from foamy foods such as bread and ice cream to foamy materials such as plant stems, bones, magma, and foam rubber. All foams have one characteristic in common: The bubbledelimiting films minimize surface energy by encapsulating the largest volume while using the least amount of material.

Hassan Aref, professor and head of the Theoretical and Applied Mechanics Department, said, "In a common liquid foam, like a soap froth, the elastic energy in the films is negligible compared with the work required to compress the air in the bubbles. The individual bubbles, which are

\section{Cost-Effective Portable Spin Coaters}

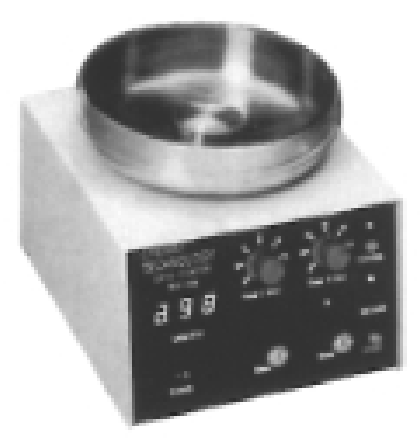

Two-Stage Spinning

- Dispense liquid during Stage 1

- Spin-up and flatten during Stage 2

\section{Adjustable Speed}

Stage 1

- 500 to $2,500 \mathrm{rpm}$

- 2 to 18 seconds

\section{Stage 2}

- 1,000 to $8,000 \mathrm{rpm}$

- 3 to 60 seconds

\section{Precision Dip Coaters}

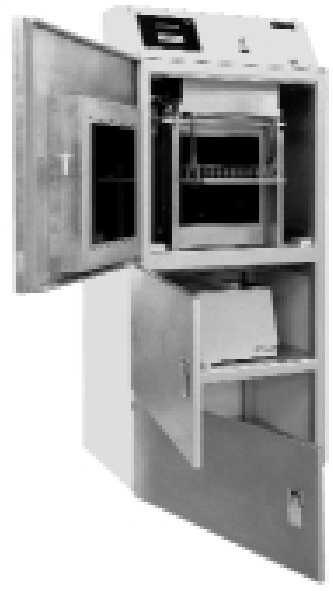

Robust Laboratory Coater

- Easy to operate

- Speed range of 0 to $30 \mathrm{~cm}$ per minute

- Adjustable travel span

- Controllable atmosphere

- Curing chamber

- Custom fabrication

\section{Coating Solutions}

- Available for metal oxides, nitrides and carbides

\section{Distributorships Available}

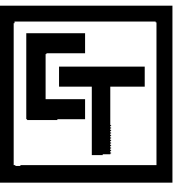
CHEMAT TECHNOLOGY, INC.

9036 Winnetka Avenue, Northridge, CA 91324

US Toll-Free: 800-475-3628 • Non-U.S. Toll-Free: 818-727-9786

Fax: 818-727-9477 • E-mail: chemat@aol.com www.chemat.com often of roughly comparable size, retain constant volumes, except for the slow redistribution of gas by diffusion or the rupturing of films between bubbles. If you imagine greatly enhancing the surface tension, however, the elastic energy in the films will compress most of the bubbles, leading to a very different structure."

To investigate this phenomenon, Aref and graduate student Dmitri Vainchtein first derived the equation of state for compressible foam. "This equation shows that foam with a free boundary will expand to a maximum volume if the external pressure is lowered at constant temperature," Aref said. "The equation also suggests that the same foam-when enclosed in a container-can be expanded further but will become unstable at a certain volume that we can predict."

Though difficult to explore experimentally, as reported in the December 1999 issue of Physics of Fluids, the nature of the instability was revealed in a series of numerical simulations. Aref said, "We observed what may be described as two 'phases' of foam. In one phase, we have a large number of small bubbles clustered together. In the other phase, we must then have a small number of much larger bubbles that occupy most of the space in the container."

The increased surface tension appears to compress most of the bubbles, forcing the remaining bubbles to expand to fill the space, Aref said. This phenomenon might provide a model for the undesirable formation of large voids in solidifying foams, including those that form when baking bread. "As bread is baked, the bubble membranes begin to harden, which may roughly correspond to an increase in surface tension," Aref said. "The resulting segregation instability results in a loaf that contains clusters of tiny bubbles embedded in a background of a few much larger bubbles."

\section{Carbon Nanotubes Exhibit Tensile Strength of $63 \mathrm{GPa}$}

Researchers at Washington University in St. Louis have found that $63 \mathrm{GPa}$ is the highest tensile-strength value of the outermost shell of a single nanotube. In an experiment performed by Rodney S. Ruoff, associate professor of physics, and his research group, individual multiwalled carbon nanotubes (MWCNT)-rolled sheets of graphite-were picked up, positioned, and firmly attached on a nanometer length scale, and tested with tension until broken.

As reported in the January 28 issue of Science, MWCNTs break with what Ruoff refers to as a "sword-in-sheath" mecha- 